\title{
EXERCÍCIO FÍSICO E MECANISMOS MOLECULARES DA CAPTAÇÃO DE GLICOSE NO DIABETES TIPO 2: REVISÃO INTEGRATIVA ${ }^{1}$
}

\author{
PHYSICAL EXERCISE AND MOLECULAR MECHANISMS OF GLUCOSE \\ UPTAKE IN TYPE 2 DIABETES: INTEGRATIVE REVIEW
}

\section{Raquel da Franca ${ }^{1}$}

\section{RESUMO}

A hiperglicemia, defeitos na produção/secreção de insulina e resistência a esse hormônio, são características fisiopatológicas observadas em indivíduos diabéticos tipo 2. O exercício físico é um aliado importante para o manejo glicêmico, porém os mecanismos envolvidos nesse processo são múltiplos, complexos e precisam ser mais explorados. O objetivo deste estudo foi analisar os mecanismos moleculares independente de insulina envolvidos no controle glicêmico em diabéticos tipo 2 em resposta ao exercício físico. Trata-se de uma revisão integrativa, com período de busca de 2000 a 2020 de artigos indexados nas bases de dados PubMed, SciELO e Medline, com os descritores encontrados no DECS e MESH. Foram investigados sujeitos diabéticos tipo 2 de ambos os sexos e com idade superior a 18 anos. Foram encontrados 632 artigos, dos quais apenas 4 ( $n=88$ ) atenderam aos critérios de inclusão. O exercício físico pode auxiliar no manejo glicêmico de diabéticos tipo 2, pois estimula vias através da contração muscular, causando aumento da expressão proteica nos tecidos, com maior destaque para a ativação da 5'-monofosfato-adenosina proteína quinase ativada e do transportador de glicose 4 nos diabéticos insulinorresistentes. Embora as evidências tenham mostrado que a ativação de algumas moléculas em resposta ao exercício físico seja benéfica para o manejo glicêmico do diabetes tipo 2, há necessidade de maior exploração desses mecanismos, pois ainda não estão bem descritos na literatura.

Palavras-chave: Diabetes tipo 2, GLUT4, Captação, Exercício físico, Quinases.

\section{ABSTRACT}

Hyperglycemia, defects in insulin production/secretion and resistance to this hormone are pathophysiological characteristics observed in type 2 diabetic individuals. Physical exercise is an important ally for glycemic management, but the mechanisms involved in this process are multiple, complex and need to be further explored. The aim of this study was to analyze the independent molecular mechanisms of insulin involved in glycemic control in type 2 diabetics in response to physical exercise. This is an integrative review, with a search period from 2000 to 2020 of articles indexed in the PubMed, SciELO and Medline databases, with the descriptors found in decs and mesh. Type 2 diabetic subjects of both sexes and older than 18 years were investigated. A total of 632 articles were found, of which only $4(n=88)$ met the inclusion criteria. Physical exercise can help in the glycemic management of type 2 diabetics, as it stimulates pathways through muscle contraction, causing increased protein expression in tissues, with greater emphasis on the activation of 5'-monophosphate-adenosine activated protein kinase and glucose transporter 4 in insulin-resistant diabetics. Although evidence has shown that the activation of some molecules in response to physical exercise is beneficial for glycemic management of type 2 diabetes, there is a need for greater exploration of these mechanisms, as they are not yet well described in the literature.

Keywords: Type 2 diabetes, GLUT4, Capture, Physical exercise, Kinases.

1 Bacharelado em Educação Física e Pós-graduanda da Residência Multiprofissional em Saúde da Família, Fundação Estatal Saúde da Família/Fundação Oswaldo Cruz, Salvador - BA, Brasil. E-mail: raquelfrancaedfisica@outlook.com 


\section{INTRODUÇÃO}

O diabetes mellitus tipo 2 (DM2) é uma síndrome metabólica caracterizada pela hiperglicemia, resistência à insulina e deficiência na secreção/produção desse hormônio. Estimativas mundiais indicam que cerca de 382 milhões de pessoas vivem com DM (8,3\%), podendo chegar em 2035 a 592 milhões (FLOR, CAMPOS, 2017). No Brasil, no ano de 2019 o número de casos de DM2 foi equivalente a 16,8 milhões de pessoas (SOCIEDADE BRASILEIRA DE DIABETES, 2019).

O aumento exponencial do DM2 está associado a fatores de risco modificáveis e não-modificáveis. As mudanças do estilo de vida podem reduzir a sua incidência em até $58 \%$ em 3 anos (MACHADO, SCHAAN, SERAPHIM, 2006). O exercício físico em conjunto ao uso de fármacos são alternativas eficazes para o manejo glicêmico no DM2 (FERRARI et al., 2019), bem como a reeducação alimentar (SANTOMAURO et al., 2008).

O exercício físico possui efeito hipoglicêmico agudo e crônico em indivíduos diabéticos independente de insulina, estimulando a captação da glicose nos tecidos adiposo e muscular, bem como alterando a sensibilidade a insulina (HOLTEN et al., 2004). Estudos recentes têm evidenciado que no pós-treinamento resistido, aeróbico, combinado (resistido em conjunto ao aeróbico na mesma ou em sessões diferentes) ou treinamento intervalado de alta intensidade (HIIT) é possível manter os valores glicêmicos regulados por até 48 horas em indivíduos diabéticos tipo 2. No entanto, para mantê-los cronicamente, é necessário que haja uma frequência semanal regular dos exercícios prescritos, não devendo ultrapassar intervalos maiores que 72 horas (LLOPIZ, GALBIS, 2015).

Um dos mecanismos fisiológicos envolvido na captação da glicose independente de insulina muito estudado atualmente é através da enzima 5' proteína quinase ativada por AMP (AMPK). Ao realizar exercícios físicos (EF), ocorre elevação da taxa AMP:ATP (adenosina difosfato: adenosina trifosfato) e a contração muscular ocasiona uma cascata de sinalizações envolvendo várias quinases e miocinas, as quais ativam a AMPK e esta, por sua vez, estimula o transportador de glicose 4 (GLUT4) a deslocar-se para a membrana plasmática, visando a captação de glicose sanguínea e sua absorção no tecido muscular, bem como adiposo (RICHTER, HARGREAVES, 2013).

Embora a literatura prévia tenha demonstrado os benefícios do exercício físico no manejo do DM2, os mecanismos moleculares que mediam a translocação GLUT4 e o aumento da absorção de glicose em resposta ao EF em humanos, ainda não estão bem descritos. Diante disso, se faz necessária maior exploração para compreender as vias envolvidas no transporte de glicose independente de insulina nesse público (STANFORD, GOODYEAR, 2014).

O objetivo desse estudo é analisar os mecanismos moleculares envolvidos na translocação do GLUT4 e captação de glicose em indivíduos portadores de diabetes tipo 2, independente de insulina e em resposta ao EF. 


\section{MATERIAIS E MÉTODOS}

Trata-se de uma revisão integrativa da literatura, com abordagem quantitativa, um tipo de estudo que consiste em seis etapas, sendo elas: 1- a identificação do tema e seleção da hipótese ou questão de pesquisa; 2- estabelecimento de critérios para inclusão e exclusão de estudos/amostragem ou busca na literatura; 3 - definição das informações a serem extraídas dos estudos selecionados/ categorização dos estudos; 4- avaliação dos estudos incluídos; 5- interpretação dos resultados; 6- apresentação da revisão/síntese do conhecimento.

Para construção da pergunta de pesquisa, foi empregada a ferramenta PICO: 1) P= população; 2) $\mathrm{I}=$ intervenção; 3) $\mathrm{C}=$ comparação; 4) $\mathrm{O}=$ desfecho (SANTOS, PIMENTA, NOBRE, 2007), onde a pergunta foi: "quais os mecanismos moleculares envolvidos na translocação do GLUT4 e captação de glicose em indivíduos portadores de DM2 em resposta ao exercício físico e independente de insulina?".

As buscas de artigos foram realizadas nas seguintes bases de dados: PubMed (National Library of Medicine), SciELO (Scientific Electronic Library Online) e MEDline (Medical Literature Analysis and Retrievel System Online). Foram aplicados os descritores "diabetes tipo 2", "GLUT4" "captação", "exercício físico" e "quinases" em português, bem como "type 2 diabetes", "GLUT4" "uptake", "physical exercise" and "kinases" em inglês e "diabetes tipo 2", "GLUT4" "captación", “ejercicio físico" y "quinasas” em espanhol, devendo constar no MESH (Medical Subject Headings) e no DECS (Descritores em Ciências da Saúde) da BIREME.

O período de buscas dos artigos ocorreu entre 2000 a 2020. Já o período de análise foi finalizado em fevereiro de 2021. Também foram empregados os operadores booleanos "AND" e "OR" para buscas mais específicas na literatura.

Os critérios de inclusão foram: estudos os quais tiveram intervenção com pessoas diabéticas tipo 2, sedentárias, com idade igual ou superior a 18 anos, de ambos os sexos e que tiveram como desfechos: 1) primário- captação de glicose mediada por uma ou mais quinases; 2) secundáriosfatores envolvidos na translocação do GLUT4 e aumento da sensibilidade a insulina. Também foram inclusos estudos que tiveram como intervenção pelo menos um tipo de exercício físico do tipo aeróbio ou anaeróbio, com intensidades baixa, moderada ou alta.

Já os critérios de exclusão foram estudos com pessoas com neuropatia diabética, gestantes diabéticas ou pesquisas que avaliaram outros desfechos, pois fugiam da proposta deste artigo.

\section{RESULTADOS E DISCUSSÃO}

Inicialmente foram identificados 632 artigos nas bases de dados. Após a aplicação dos filtros, avaliação de duplicatas e leitura de título e resumo, foram excluídos 559 artigos. Depois da leitura completa dos estudos elegíveis, apenas 4 artigos $(n=88)$ atenderam aos critérios de elegibilidade deste 
estudo e foram incluídos. Já os outros excluídos continham: 21 artigos incluíam gestantes diabéticas, 31 tiveram outros desfechos e 17 tiveram como eixo da pesquisa a neuropatia diabética.

Figura 1 - Fluxograma das estratégias de buscas para identificação dos artigos elegíveis.

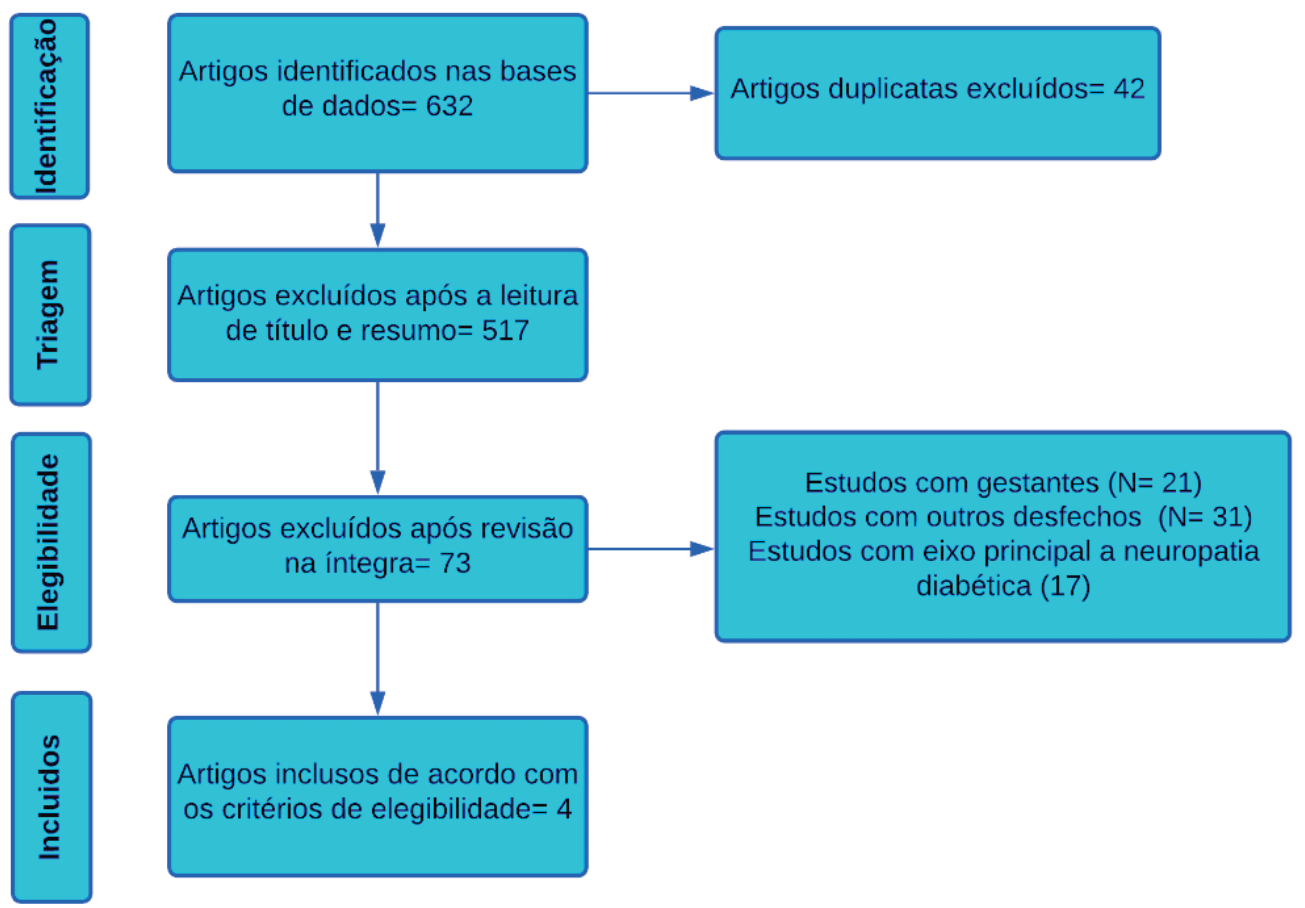

Fonte: a autora (2020).

Já na tabela 1. consta a relação de moléculas encontradas durante as buscas, envolvidas no processo de captação de glicose e de sensibilidade a insulina no DM2. Logo em seguida, na tabela 2. contém os dados referentes as características dos estudos e das amostras, bem como o nome do autor, ano, tipo de estudo e principais resultados.

Tabela 1 - Relação das moléculas envolvidas no processo de captação da glicose e aumento da sensibilidade a insulina em resposta ao exercício físico.

\begin{tabular}{ll}
\hline \multicolumn{1}{c}{ ABREVIAÇÃO } & \\
\hline GLUT4 & Transportador de glicose 4 \\
\hline AMPK & 5' proteína quinase ativada por AMP \\
\hline PGC1- $\alpha$ & Coativador-1 $\alpha$ do receptor ativado por proliferador do peroxissoma \\
\hline LKB1 & Quinase hepática B1 \\
p38 MAPK ou HDACS & 38' proteína quinase ativada por mitógenos ou histonas deacetilases \\
\hline CaMKK & Quinase dependente de cálcio/calmodulina \\
\hline Aktlou PKB $\alpha$ & Proteína quinase serina/treonina 1 \\
\hline Akt2 ou PKB $\beta$ & Proteína quinase serina/treonina 2 \\
\hline GS & Enzima glicogênio sintase \\
\hline TBC1D4 ou AS160 & Proteína Rac1, TBC1 membro da família de domínio 4, substrato AKT de 160 kDa \\
TBC1D1 & Proteína Rac1, TBC1 membro da família de domínio 1 \\
Hexokinase II & Enzima da via glicolítica que converte a glicose em glicose-6-fosfato \\
\hline IL-15 & Miocina interleucina-15 \\
\hline IL-6 & Miocina interleucina-6 \\
\hline
\end{tabular}




\begin{tabular}{ll} 
PKC & Proteína quinase C \\
\hline eNOS & Enzima óxido nítrico sintase endotelial \\
\hline MEF2A & Proteína fator melhorador específico do miócito 2A \\
\hline AICAR & $\mathbf{5 - a m i n o i m i d a z o l - 4 - c a r b o x a m i d a - 1 - \beta - D - r i b o f u r a n o s i ́ d e o ~}$ \\
\hline AMP & Adenosina difosfato \\
\hline ATP & Adenosina trifosfato \\
\hline
\end{tabular}

Fonte: a autora (2021).

Tabela 2 - Características dos estudos e participantes inclusos no trabalho, bem como os resultados encontrados.

\section{AUTOR CARACTERÍSTICA DO ESTUDO \\ E ANO E AMOSTRA \\ CARACTERÍSTICAS DAS IN- TERVERNCÕES \\ PRINCIPAIS RESULTADOS}

Holten et $\quad$ ECR com 17 indivíduos, todos do sexo

al. (2004) M: G1= grupo intervenção com 10 pessoas com DM2 e tempo de duração da doença de 2-11 anos. G2= 7 sujeitos saudáveis do grupo controle. As médias de idade no G1 e G2, respectivamente, foram: $62 \pm 2$ e $61 \pm 2$ anos.

Vind et al. $\quad$ EC com 26 indivíduos do sexo M, (2010) obesos e sedentários divididos em grupos: G1= o grupo de treinamento com 13 obesos diabéticos tipo 2 e $\mathrm{G} 2=\mathrm{o}$ grupo controle com 13 obesos sem DM2. Média de idade dos participantes do $\mathrm{G} 1=52.8 \pm 1.3$ e do $\mathrm{G} 2=$ $52.7 \pm 1.6$

EC com 18 sujeitos: G1= com 9 pesal. (2012) soas com DM2 e tempo de duração mínimo de 1 ano, sendo 6 do sexo $\mathrm{M}$ e $3 \mathrm{~F} ; \mathrm{G} 2=$ controle sem DM2, com 9 pessoas, sendo $5 \mathrm{M}$ e $4 \mathrm{~F}$. Média de idade no $\mathrm{G} 1=53 \pm 4$ anos e no $\mathrm{G} 2=$ $48 \pm 3$.

Kjøbs-

ted et al.

(2016)
ECNA com 27 indivíduos com sobrepeso/obesidade, todos do sexo $\mathrm{M}$ : G1= 13 com DM2 e G2= 14 sem DM2. Os 2 grupos realizaram ensaios de grampo separados (dia de linha de base e dia de exercício). Média de idade dos participantes: $55 \pm 2$ anos em ambos os grupos.
Ambos os grupos realizaram uma única sessão de EA, em um cilcoergômetro durante $60 \mathrm{~min}$ a $55 \%$ da potência máxima (estimada em watts).

Os participantes exercitaram-se por 60 minutos em um ciclo ergômetro (EA) a uma intensidade de $70 \%$ de VO2max, durante 4-8 semanas. Todos foram avaliados tanto no repouso, quanto após $4 \mathrm{~h}$ de realização de atividade aeróbica.
Foi observado aumento de $40 \%$ da densidade de GLUT4 após ER no MI treinado $(P<0,05)$ do G1, mas no G2 o valor não foi estatisticamente significante. Houve aumento significativo no teor proteico do receptor de insulina do $\mathrm{G} 2=19 \pm 7 \%$; já no $\mathrm{Gl}=21 \pm 6 \%$, ambos com $\mathrm{P}<0,05$. Houve aumento nos Akt 1 e 2 , teor de proteína e atividade total de GS em ambos $(P<0,05)$.

O EA aumentou os níveis de GLUT4 em 20\% no G2 e $30 \%$ no G1 e da hexokinase II em $45 \%$ no G2 e $75 \%$ no G1, com redução no pré e pós treinamento pra ambos $(p<0,05)$. Houve aumento significativo na fosforilação da AMPK ( $p=0,003)$, Akt1 em 20\% no G2 e 50\% no G1; já a Akt2 foi aumentada em 30-40\% em ambos os grupos. Houve melhorias na SI em ambos (cerca de $20 \%$ ), associadas ao aumento de Akt, TBC1D4, AMPK, SG, hexokinase II e GLUT4 (20-75\%).

A expressão do GLUT4 aumentou $(\mathrm{p}<0,05)$ em uma medida semelhante imediatamente após o EA em G2 $(60 \%)$ e G1 $(66 \%)$ e permaneceu elevado $(p<0,05)$ PE (3h), sem diferenças entre os grupos. Houve aumento na expressão de p38MAPK e PGC-1 $\alpha(p<0,05)$ PE, não sendo diferentes entre os grupos. A AMPK foi aumentada $(\mathrm{p}<0,05)$ imediatamente PE em $135 \%$ e $149 \%$ no G2 e G1, respectivamente.

Aumento mais acentuado na fosforilação de TBC1D1 no G1, do que G2 durante a recuperação do exercício $(P<0,01)$. A atividade total de AMPK aumentou cerca de 1,3 vezes em resposta ao exercício $(P<0,05)$ e permaneceu elevada por até $3 \mathrm{~h}$ em recuperação, quando comparada com o pré-exercício $(P<0,05)$. Não foram encontradas diferenças significativas na atividade total de AMPK entre indivíduos do G1 e G2 em ambos os dias (linha de base e de exercício).

Legenda: $\mathrm{TA}=$ treinamento aeróbio; $\mathrm{VS}=$ volume por sessão; $\mathrm{FS}=$ frequência semanal; $\mathrm{SI}=$ sensibilidade insulínica. $\mathrm{M}=$ masculino e $\mathrm{F}=$ feminina; $\mathrm{VO} 2 \mathrm{max}=$ consumo máximo de oxigênio; $\mathrm{EA}=$ exercício aeróbico; $\mathrm{ER}=$ exercício resistido; $\mathrm{DM} 2=$ diabetes mellitus tipo 2; $\mathrm{Gl}=$ grupo $1 ; \mathrm{G} 2=$ grupo $2 ; \mathrm{PG}=$ progressão gradual; $\mathrm{PE}=$ pós-exercício; $\mathrm{R}=$ repetições; $\mathrm{S}=$ séries; $\mathrm{MI}=$ membro inferior; $1 \mathrm{RM}=$ uma repetição máxima; GLUT4= transportador de glicose 4; AMPK= 5' proteina ativada por adenosina difosfato; p38MAPK=38' proteína quinase ativada por mitógenos; PGC-1 $\alpha=$ Coativador-1 $\alpha$ do receptor ativado por proliferador do peroxissoma; TBC1D1= proteína Rac1,

TBC1 membro da família de domínio 1; TBC1D4= proteína Rac1, TBC1 membro da família de domínio 4; SG= glicogênio sintase;

hexokinase II= enzima glicolítica conversora de glicose; Aktl= quinase serina/treonina 1; Akt2= quinase serina/treonina 2;

$\mathrm{ECR}=$ ensaio clínico randomizado; $\mathrm{EC}=$ ensaio clínico; $\mathrm{ECNA}=$ ensaio clínico não-aleatorizado; $\pm=$ desvio padrão. 
Os resultados deste estudo demonstram que os mecanismos envolvidos na captação de glicose em pessoas com DM2 em resposta ao EF são múltiplos e complexos. Em ambos os estudos houve aumento na expressão do GLUT4 mediados por diferentes genes, com maior presença de ativação da via AMPK (FERRARI et al., 2019).

De acordo com Wang, Simar e Singh (2009) outros mecanismos encontrados que podem estar diretamente relacionados a translocação de GLUT4 e absorção de glicose intracelular são o aumento da atividade da enzima óxido nítrico sintase endotelial (eNOS), a ativação das proteínas cinase ativada por mitógenos (MAPK), cinase dependente de cálcio/calmodulina (CaMK), proteína quinase C (PKC), elevação de cálcio $(\mathrm{Ca}++)$ no interior celular ou até mesmo a hipóxia.

Corroborando com este dado, o estudo de Vind et al. (2011) avaliou 26 indivíduos obesos separados por grupo controle (sem DM2) e grupo experimental (com DM2), os quais realizaram EA durante 10 semanas, com máximo de 35 minutos por sessão e intensidade em $60 \%$ do VO2máx. Após a intervenção, esses indivíduos tiveram aumento em cerca de $20 \%$ na sensibilidade a insulina à medida em que o teor proteico das quinases Akt1, Akt2, GS, TBC1D4 (substrato Akt de 160 ou AS160), GLUT4, hexokinase II e AMPK também se elevaram, em cerca de 20-70\%.

Já Hussey et al. (2012) demonstraram que apenas uma única sessão de EF é suficiente para induzir a expressão desses genes metabólicos. Em pacientes diabéticos a expressão genética do coativador transcricional que regula os genes envolvidos no metabolismo energético ( $P G C-1 \alpha)$ e é mediado pela quinase p38 MAPK, aumentou cerca de 6,5 vezes 3 horas pós exercício, além de ter aumentado em até $66 \%$ a expressão do GLUT4.

Ainda nessa perspectiva, 27 indivíduos com sobrepeso/obesidade do sexo masculino, participaram de um ensaio clínico, no qual eles foram separados em G1 e G2, intercalando dias de linha de base e dias de EF. No dia do exercício, a fosforilação de TBC1D1 com exercícios semelhantes nos dois grupos foi aumentada em cerca 1,8 vezes $(p<0,01)$. A atividade total de AMPK aumentou cerca de 1,3 vezes em resposta ao exercício $(p<0,05)$ e permaneceu elevada por até $3 \mathrm{~h}$ em recuperação, quando comparada com o pré-exercício $(p<0,05)$. Não foram encontradas diferenças significativas na atividade total de AMPK entre indivíduos do G1 e G2 em ambos os dias, linha de base e de exercício (KJOBSTED et al., 2018).

No entanto, o estudo anterior não descreveu a frequência semanal com que esses indivíduos realizaram os EF, sendo este um parâmetro fundamental para compreender os mecanismos envolvidos na regulação de glicose. Isto porque o controle glicêmico se mantém por até 48 horas após estímulo do EF. Dessa forma, o diabético tipo 2 deve manter frequência semanal constante de EF e não ultrapassar 72 sem praticá-lo (LLOPIZ, GALBIS, 2015).

Estas evidências demonstram que independentemente do tipo de exercício físico praticado, há uma relação grande entre esta prática e aumento na translocação de GLUT4 nos tecidos e maior ativação através da AMPK. No tecido muscular, a enzima AMPK é capaz de aumentar a oxidação 
lipídica e adaptar a ressíntese de glicogênio em resposta ao EF (JEON, 2016). Em modelos transgênicos, foi observado que roedores com DM2, os quais realizaram EF aeróbicos durante 16-20 semanas, tiveram uma melhora na oxidação lipídica durante a intervenção. No entanto, não houve associação entre a melhora dessa oxidação nos lipídios com o controle da glicose muscular in vivo (JEPESSEN et al., 2013).

Já no tecido adiposo, algumas miocinas como a interleucina-15 (IL-15) e interleucina-6 (IL-6) conseguem estimular o aumento da expressão de GLUT4, ativar a AMPK, a qual transloca o GLUT4 para a membrana da célula, podendo promover a captação de glicose induzida pela contração muscular (FUJIMOTO, 2019).

Além da absorção de glicose, o aumento na sensibilidade à insulina pode estar associado a melhor sinalização da TBC1D4 (fosforilada pela AMPK), bem como incrementos nos níveis de proteínas no músculo esquelético. Não obstante, independente do período, se agudo ou crônico, o EF aumenta a expressão de algumas dessas proteínas no músculo esquelético de humanos, principalmente a AMPK e TBC1D4 (CARTEE, 2015).

Outra proteína envolvida no transporte de glicose muscular muito estudada atualmente é a quinase hepática B1 (LKB1), haja vista que esta auxilia na fosforilação da enzima AMPK em resposta ao EF. O teor de proteína LKB1 aumenta simultaneamente a elevação da expressão do PGC1 no músculo (SRIWIJITKAMOL, IVY, ROBERTS, 2006). Em contrapartida, foi percebido que a exclusão específica dessa quinase em indivíduos com DM2, inibiu parcialmente a translocação da glicose nos tecidos (KOH, BRANDAUER, GOODYEAR, 2008).

Ao que tudo indica, não somente pessoas obesas e com DM2 tem maior ativação da via AMPK em resposta ao EF; as evidências atuais também sugerem que há essa relação entre sujeitos saudáveis. Em vista disso, um estudo dividiu em grupos de intervenção onde o grupo 1 (G1) realizou EF durante 90 min e pico de 67\% VO2, o grupo 2 (G2) com 20 min com VO2 de 80\%, o grupo 3 (G3) com 2 min e 110\% do VO2máx (G3=9) e o grupo 4 (G4) um sprint máximo com 30 segundos de duração. No G1 a fosforilação de AS160 aumentou em 1 hora $(60 \%, \mathrm{P}=0,06)$ e permaneceu até 90 minutos de EF $(120 \%, \mathrm{P}<0,05)$. Já a atividade de uma das isoformas catalíticas da AMPK (quinase de proteína ativada alfa2beta2gamma3-AMP) aumentou significativamente para um nível de estado estável após $30 \mathrm{~min}$, enquanto a atividade da outra subunidade (alfa2beta2gamma1-AMP) aumentou após 1 hora de exercício com um aumento ainda maior após 90 min, demonstrando que quanto maior a duração do EF, maior o estímulo da AMPK e AS160. Nos G2, G3 e G4, não houve aumento das atividades de alfa2beta2gamma3-AMPK, fosforilação de AS160 e nem alfa2beta2gamma1-AMPK (TREEBAK et al., 2007).

Todavia, um outro estudo demonstrou que indivíduos com obesidade e DM2 que realizaram intervenções de EA com intensidades baixa (50\% do VO2máx) e moderada (70\% do VO2máx), obtiveram pouco estímulo do eixo AMPK-AS160 (TREEBAK et al., 2007). Já em outro ensaio, foi mostrado que após 6 meses de intervenção, tanto os EF de baixa, quanto moderada intensidade cau- 
saram efeitos eficazes na HbAlc, no colesterol e peso corporal de 37 indivíduos com o mesmo perfil patológico (HANSEN et al., 2009).

Outros parâmetros avaliados nestes estudos e que podem ter influenciado no controle glicêmico do DM2, foram a idade e sexo dos participantes. A maioria pertenceu ao sexo masculino (94\%), com idade superior a 40 anos, evidenciando que os jovens adultos estão cada vez mais precocemente sendo atingidos pela doença e a prevalência em pessoas do sexo masculino tem aumentado. Não obstante, as amostras foram pequenas e heterogêneas, o que pode ter influenciado nos resultados do estudo e aumentado o risco de viés (EARNEST et al., 2014).

Um trabalho recente com modelo transgênico evidenciou que as quinases PAKs do grupo I (akt1) e grupo II (akt2) são ativadas após o EF, contribuindo para a regulação do transporte de glicose em ratos, porém não são essenciais nesse processo, havendo ainda uma menor contribuição por parte da akt1 quando comparada a akt2 (MOLLER et al., 2020).

Há algumas décadas, buscando compreender estes mecanismos envolvidos no controle glicêmico do DM2, a maior parte dos estudos abordava o EA no DM2. No entanto, os estudos atuais tem cada vez mais buscado entender como outros tipos de exercícios como o resistido, combinado e HIIT atuam nesse sistema (AKOBENG, 2008).

Em vista disso, buscando compreender os efeitos do ER em diabéticos tipo 2, um estudo avaliou os efeitos de uma intervenção de 6 semanas com 3 exercícios para apenas um membro inferior, frequência semanal de 3x, volume de 30 minutos por sessão e intensidade estimada em $50-80 \%$ de 1RM nesse público. Ao final, perceberam que houve aumento de 40\% da densidade de GLUT4 após ER no MI treinado $(P<0,05)$ desse grupo. Além disso, o teor proteico do receptor de insulina aumentou significativamente $(19 \pm 7 \%)$ (CIOLAC, GUIMAR ÃES, 2004).

Embora os estudos tenham avançado com o passar dos anos, explorando e buscando compreender os mecanismos bioquímicos envolvidos na regulação da glicose sanguínea no DM2, a via AMPK parece ser a mais presente na translocação do GLUT4 e na captação da glicose sanguínea em resposta ao EF (FERRARI et al., 2019).

Durante a realização de exercícios físicos, a atividade contrátil dos músculos esqueléticos altera o estado de energia celular, resultando em um aumento na exigência metabólica corporal e elevação da taxa AMP:ATP. Assim, a AMPK é fosforilada após uma cascata de sinalização mediada por outras proteínas e, após a fosforilação, a AMPK aciona vias que geram o aumento do ATP, promovendo a translocação de vesículas contendo GLUT4 para a membrana plasmática.

Ao ser translocado, o GLUT4 capta a glicose circulante no sangue e a transporta para o interior celular através de difusão facilitada. Esta molécula de glicose é convertida em ATP no meio intracelular e acaba gerando energia. Dessa forma, as moléculas de glicose sanguínea são reduzidas, mantendo o controle glicêmico agudo por até 48 horas. Abaixo segue a Figura 2 com uma esquematização sobre esse processo. 
Figura 2 - Esquema sobre a captação de glicose via AMPK, em resposta ao exercício físico. Ao realizar o exercício ocorrem: 1) contrações musculares concêntricas e excêntricas 2) deslizamento entre os filamentos de actina e miosina no músculo esquelético; 3) decréscimo energético, elevação na taxa AMP:ATP (adenosina monofosfato: adenosina trifosfato); 4) cascata de sinalização intracelular envolvendo várias proteínas como CaMKK: Quinase dependente de cálcio/calmodulina, AICAR: 5-aminoimidazol-4-carboxamida-1- $\beta$-D-ribofuranosídeo, MEF2a: Proteína fator melhorador específico do miócito 2A, HDACS: histonas deacetilases, AS160: substrato AKT de 160 kDa e p38MAPK:

38 ' proteína quinase ativada por mitógenos. Em seguida, essa cascata provoca a fosforilação da AMPK e a sua ativação estimula o transportador de glicose 4 (GLUT4) a se translocar para a membrana plasmática, o qual capta a glicose sanguínea e a transporta para o meio intracelular através de difusão facilitada, mantendo assim o controle glicêmico no diabetes tipo 2 (DM2).

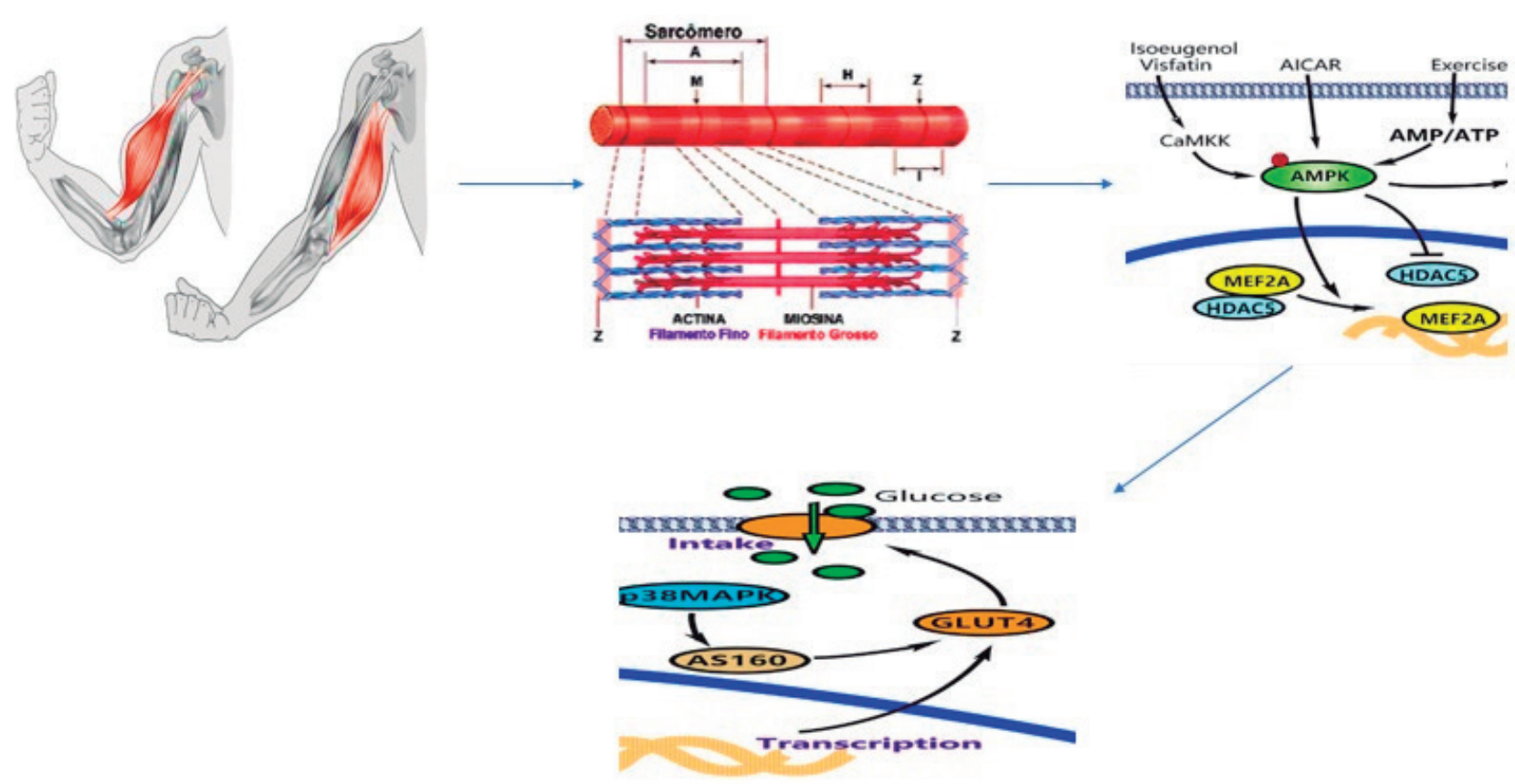

Fonte: a autora (2021), adaptado de Richter e Hargreaves (2013).

O manejo glicêmico sanguíneo em indivíduos com DM2, pode diminuir os riscos de complicações micro e macro vasculares decorrentes da síndrome metabólica (PAULI et al., 2009). Alguns estudos têm mostrado que a desregulação glicêmica pode estar associada a fatores como obesidade e excesso de disponibilidade lipídica, pois estes causam perda da sensibilidade a insulina e aumentam a resistência a esse hormônio no músculo esquelético em humanos (BIRD, HAWLEY, 2017). Nesse sentido, os resultados deste estudo mostraram que boa parte dos indivíduos que tinham DM2 também eram obesos, sendo que a obesidade, resistência a insulina e sedentarismo aumentam a predisposição de DM2 e doenças cardiovasculares (PEHMOLLER et al., 2012).

Uma das formas de diminuição da resistência a insulina e aumento da captação glicêmica é através do $\mathrm{EF}$, pois indivíduos diabéticos insulinoresistentes que mantiveram um programa estruturado de EF, conseguiram manejar a glicemia melhor do que indivíduos sedentários (WANG, SIMAR, SINGH, 2009). Evidências demonstram que adultos com DM2 que aderem a programas de EF a longo prazo, conseguem diminuir o percentual de HbAlc. Isso se deve ao aumento da absorção de glicose por meio do GLUT4, o qual é ativado pela AMPK via contração muscular prolongada (FERRARI et al., 2019). 
O aumento do recrutamento de GLUT4 para a membrana plasmática após uma sessão de EF também está associado ao aumento da sensibilidade à insulina nos tecidos. A diminuição da expressão dessa proteína tem relação direta com a prevalência de resistência a esse hormônio. Para prevenção dessa condição de saúde, alguns autores destacam a importância do EF associada a presença da TBC1D4 e GS induzidas por lipídios nos tecidos (SANTOS et al., 2015).

Corroborando com o estudo anterior, foram analisadas pessoas diabéticas insulinorresistentes, as quais foram divididas em grupos com: exercício aeróbio (EA), exercício resistido (ER) e exercício combinado (EC), visando observar os efeitos hipoglicemiante do EF, com duração de 38 semanas. Foram aferidas 322 glicemias do grupo EA, 258 do ER e 259 do EC, sendo observado no geral 19 moderadas (50-70 mg/dl), 214 leves (70-100 mg/dl) e nenhum grave Foi percebido que o EA teve maior efeito hipoglicemiante no pré-teste, quando comparado aos outros grupos (SUH, PAIK, JACOBS, 2007).

Já em outro estudo mais recente, foi percebido que os ER ou EC são mais eficientes na diminuição da glicemia em comparação ao EF aeróbico. Isso se deve ao tipo de fibra muscular recrutado no EA, que são fibras oxidativas do tipo 1, as quais ativam mais facilmente o GLUT4 e aumentam o efeito hipoglicemiante pós exercício. Com relação ao ER e EC há pouca diferença nesse processo, havendo ainda menor efeito no ER, evidenciando que para o DM2 o mais adequado seria o ER ou EC por reduzirem esse efeito pós-exercício e promoverem hipertrofia, força, resistência e potência muscular (BRITO et al., 2019).

Além disso, o ER consegue promover aumento da sensibilidade nos receptores da insulina (MARQUES, PIGOSO, 2016). No entanto, além do ER, o EA aumenta a sensibilidade à esse hormônio, sendo que os mecanismos envolvidos nesta ação parecem ser divergentes, o que sugere que a combinação das duas modalidades (EC) pode ser mais benéfica quando comparada ao exercício isolado (CIOLAC, GUIMARÃES, 2004).

Sabe-se que o exercício físico tem efeitos agudos e crônicos sobre a glicemia no DM2 (CARTEE et al., 2015). Em vista disso, foi realizado um estudo com humanos no qual os efeitos agudos diminuíram 78\% da glicemia capilar com uma variação de 0,4 até $62,5 \%$ em relação ao pré-exercício e uma redução média da glicemia pós exercícios em 18\% $(\mathrm{p}<0,05)$, demonstrando a capacidade de efeito hipoglicemiante do EF (CAMBRI et al., 2007).

Já em modelos transgênicos, uma intervenção com ratos machos em condições de jejum, com exercício aeróbico, volume de 180 minutos, em uma esteira com inclinação de $15 \%$, evidenciou que essa única sessão de exercício foi capaz de aumentar a absorção de glicose nos músculos de contrações lenta (fibra tipo II) e rápida (I) por várias horas (IWABE et al., 2014).

Com relação aos efeitos crônicos do EF, 24 indivíduos sedentários de ambos os sexos e com DM2, média de idade de 60,41 \pm 7,87, participaram de intervenções de EC e EA durante 20 semanas, com frequência equivalente a 3 sessões semanais. Ambos os exercícios causaram efeitos 
positivos no controle glicêmico; porém, o treinamento combinado foi mais eficaz quando comparado ao aeróbio (MORO et al., 2012).

Não obstante, outro estudo o qual envolveu 14 pessoas com DM2, um grupo com 8 desses sujeitos foi submetido a intervenção com treinamento intervalado de alta intensidade (HIIT) por 2 semanas, onde treinaram em um ergômetro, com frequência semanal equivalente a 3x. Cada sessão consistiu em 10×60 segundos de ciclismo a 90\% de esforço máximo com 60 segundos de recuperação. Ao final do ensaio, observaram um aumento acentuado na expressão GLUT4 do músculo esquelético e uma diminuição significativa nas concentrações de glicose sanguínea, mostrando que o HIIT pode ser uma estratégia eficaz para o tratamento do diabetes insulinorresistente (STANFORD, GOODYEAR, 2014), pois o aumento na expressão de GLUT4 no músculo esquelético e tecido adiposo indicam maior absorção de glicose sanguínea e menor resistência à insulina(BRITO et al., 2019).

\section{CONCLUSÃO}

Este estudo mostrou que o exercício físico pode auxiliar no manejo glicêmico de diabéticos tipo 2, pois estimula e ativa vias através da contração muscular, as quais induzem a translocação do GLUT4 e captação de glicose nos tecidos, independentes de insulina.

De acordo com os resultados, a via AMPK foi uma das principais neste processo, sendo que o GLUT4 é capaz de auxiliar não somente na absorção de glicose, mas também no aumento da sensibilidade à insulina. Entretanto, a maioria dos estudos relacionados a ativação de vias moleculares envolvidas na captação de glicose e diminuição da resistência à insulina tem estudado modelos transgênicos com ratos, sendo necessários maiores ensaios clínicos com humanos portadores de DM2, para possibilitar melhor compreensão sobre esses mecanismos, pois ainda não estão bem descritos na literatura.

\section{REFERÊNCIAS}

AKOBENG, A. K. Assessing the validity of clinical trials. Journal of Pediatriatric Gastroenterology and Nutrittion, v. 47, n. 3, p. 277-288, 2008.

BIRD, S. R; HAWLEY J. A. Update on the effects of physical activity on insulin sensitivity in humans. Open Sport \& Exercise Medicine, v. 2, n. 1, p. 000143, 2017.

BRITO, A. S. et al. Efeitos do treinamento aeróbio, resistido e combinado, sobre a hiperglicemia em jejum de diabéticos tipo 2. Ensaios e Ciência, v. 23, n. 2, p. 109-114, 2019. 
CAMBRI, L.T. et al. Efeito agudo crônico do exercício físico no perfil glicêmico e lipídico em diabéticos tipo 2. Motriz, v. 13, n. 4, p. 238-248, 2007.

CARTEE, G. D. AMPK-TBC1D4-Dependent Mechanism for Increasing Insulin Sensitivity of Skeletal Muscle. Diabetes, v. 64, n. 6, p. 1901-1903, 2015.

CIOLAC, E. G; GUIMARÃES, G.V. Exercício físico e síndrome metabólica. Revista Brasileira de Medicina do Esporte, v. 10, n. 4, p. 319-324, 2004.

EARNEST, C. P. et al. Aerobic and strength training in concomitant metabolic syndrome and type 2 diabetes. Medicine \& Science in Sports \& Exercise, v. 46, n. 7, p. 1293-1301, 2014.

FERRARI, F. et al. Review article biochemical and molecular mechanisms of glucose uptake stimulated by physical exercise in insulin resistance state: role of inflammation. Arquivos Brasileiros de Cardiologia, v. 113, n. 6, p. 1139-1148, 2019.

FERREIRA, J. S. M. N. et al. Hipoglicemia em diabéticos tipo 2 praticantes de exercício físico. ConScientiae, v. 16, n. 1, p. 58-64, 2017.

FLOR L.S, CAMPOS M.R. Prevalência de diabetes mellitus e fatores associados na população adulta brasileira: evidências de um inquérito de base populacional. Revista Brasileira de Epidemiologia, v. 20, n. 1, p. 16-29, 2017.

FUJIMOTO, T. et al. Overexpression of Interleukin-15 exhibits improved glucose tolerance and promotes GLUT4 translocation via AMP-Activated protein kinase pathway in skeletal muscle. Biomechal and Biophysical Research Communications, v. 509, n. 4, p. 994-1000, 2019.

HANSEN, D. et al. Continuous low- to moderate-intensity exercise training is as effective as moderate- to high-intensity exercise training at lowering blood $\mathrm{HbA}(1 \mathrm{c})$ in obese type 2 diabetes patients. Diabetologia, v. 52, n. 9, p. 1789-97, 2009.

HARDIE, D.G. Minireview: the AMP-activated protein kinase cascade: the key sensor of cellular energy status. Endocrinology, v. 144, n. 12, p. 5179-5183, 2003. 
HOLTEN, M.K, et al. Strength training increases insulin-mediated glucose uptake, GLUT4 content, and insulin signaling in skeletal muscle in patients with type 2 diabetes. Diabetes, v. 53, n. 2, p. 294-305, 2004.

HUSSEY, S. E. et al. Exercise increases skeletal muscle GLUT4 gene expression in patients with type 2 diabetes. Diabetes, Obesity and Metabolism, v. 14, n. 8, p. 768-771, 2012.

IVERSEN, N. et al. Mitochondrial biogenesis and angiogenesis in skeletal muscle of the elderly. Experimental Gerontology, v. 46, n. 8, p. 670-678, 2011.

IWABE, M. et al. Increased postexercise insulin sensitivity is accompanied by increased AS160 phosphorylation in slow-twitch soleus muscle. Physiological Reports, v. 2, n. 12, p. 12162, 2014.

JEON, S.M. Regulation and function of AMPK in physiology and diseases. Experimental e Molecular Medicine, v. 48, n. 7, p. e245, 2016.

JEPESSEN, J. et al. LKB1 Regulates lipid oxidation during exercise independently of AMPK. Diabetes, v. 2, n. 5, p. 1490-1499, 2013.

KJOBSTED, R. et al. AMPK in skeletal muscle function and metabolism. Federation of American Societies for Experimental Biology, v. 32, n. 4, p. 1741-1777, 2018.

KOH, H. J; BRANDAUER, J; GOODYEAR, L. J. LKB1 and AMPK and the regulation of skeletal muscle metabolismo. Current Opinion in Clinical Nutrition and Metabolic Care, v. 11, n. 3, p. 227-232, 2008.

LLOPIZ P. Q, GALBIS R. G. M. Control glucémico através del ejercicio físico en pacientes con diabetes mellitus tipo 2; revisión sistemática. Nutricion Hospitalaria, v. 31, n. 4, p. 1465-1472, 2015.

MACHADO U. F, SCHAAN, B. D; SERAPHIM, P. M. Transportadores de Glicose na Síndrome Metabólica. Arquivos Brasileiros de Endocrinologia \& Metabologia, v. 50, n. 2, p. 177-189, 2006.

MARQUES, L. F. F; PIGOSO, A. A. O treinamento de força para diabéticos tipo 2. Revista Saúde em Foco, v. 3, n. 1, p. 36-45, 2016. 
MOLLER, L. L. V. et al. The p21-activated kinase 2 (PAK2), but not PAK1, regulates contraction-stimulated skeletal muscle glucose transport. Physiological Reports, v. 8, n. 12, p. e14460, 2020.

MORO, A. R. P. et al. Efeito do treinamento combinado e aeróbio no controle glicêmico no diabetes tipo 2. Fisioterapia em Movimento, v. 25, n. 2, p. 399-409, 2012.

PAULI, J. R. et al. Novos mecanismos pelos quais o exercício físico melhora a resistência à insulina no músculo esquelético. Arquivos Brasileiros de Endocrinologia \& Metabologia, v. 53, n. 4, p. 399-408, 2009.

PEHMOLLER, C. et al. Exercise alleviates lipid-induced insulin resistance in human skeletal muscle-signaling interaction at the level of TBC1 domain family member 4. Diabetes, v. 61, n. 11, p. 2743-1752, 2012.

RICHTER E. A; HARGREAVES M. Exercise, GLUT4, and skeletal muscle glucose uptake. Physiology Journals, v. 93, n. 3, p. 993-1017, 2013.

ROCKL K. S, WITCZACK C. A, GOODYEAR L. J. Signaling mechanisms in skeletal muscle: acute responses and chronic adaptations to exercise. International Union of Biochemistry and Molecular Biology Life, v. 60, n. 3, p. 145-53, 2008.

SANTOMAURO, A. C. J. et al. Metformina e AMPK: Um antigo fármaco e uma nova enzima no contexto da síndrome metabólica. Arquivos Brasileiros de Endocrinologia \& Metabologia, v. 52, n. 1, p. 120-125, 2008.

SANTOS, C. M. C; PIMENTA, C. A. M. P; NOBRE M. R. C. The PICO strategy for the research question construction and evidence search. Revista Latino-Americana de Enfermagem, v. 15, n. 3, p. 508-511, 2007.

SANTOS, J. M. et al. The effect of exercise on skeletal muscle glucose uptake in type 2 diabetes: An epigenetic perspective. Metabolism, v. 64, n. 12, p. 1619-1628, 2015.

SOCIEDADE BRASILEIRA DE DIABETES. Diretrizes da Sociedade Brasileira de Diabetes 2019-2020. São Paulo: Clannad; 2019. 
SUH, S. H; PAIK, I. Y; JACOBS, K. Regulation of blood glucose homeostasis during prolonged exercise. Mol Cells, v. 13, n. 3, p. 272-279, 2007.

SRIWIJITKAMOL, A; IVY, J. L; ROBERTS, C. C. LKB1-AMPK sinalizando em músculos de ratos Zucker resistentes à insulina obesa e efeitos do treinamento. American Journal of Physiology-Endocrinol and Metabolism, v. 290, n. 1, p. 925-932, 2006.

STANFORD K. I; GOODYEAR L. J. Exercise and type 2 diabetes: molecular mechanisms regulating glucose uptake in skeletal muscle. Advances in Physiology Education, v. 38, n. 4, p. 308-314, 2014.

TREEBAK, J. T. et al. AS160 phosphorylation is associated with activation of alpha2beta2gamma1but not alpha2beta2gamma3-AMPK trimeric complex in skeletal muscle during exercise in humans. American Journal of Physiology-Endocrinol and Metabolism, v. 292, n. 3, p. 715-722, 2007.

VIND, B. F. et al. Impaired insulin-induced site-specific phosphorylation of TBC1 domain family, member 4 (TBC1D4) in skeletal muscle of type 2 diabetes patients is restored by endurance exercise-training. Diabetologia, v. 54, n. 1, p. 157-167, 2011.

WANG, Y; SIMAR, D; SINGH, M. A. F. Adaptations to exercise training within skeletal muscle in adults with type 2 diabetes or impaired glucose tolerance: a systematic review. Diabetes Metabolism Research and Reviews, v. 25, n. 1, p. 13-40, 2009. 
